\title{
The cadherin superfamily ${ }^{*}$
}

\author{
Jonathan Pettitt ${ }^{\S}$, Cell and Developmental Biology Program, School of \\ Medical Sciences, University of Aberdeen, Institute of Medical Sciences, \\ Aberdeen AB25 2ZD UK
}

\section{Table of Contents}

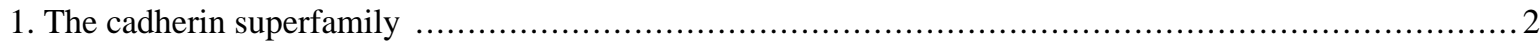

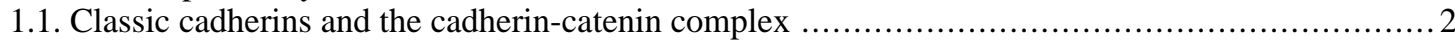

1.2. The $C$. elegans cadherin-catenin complex .................................................... 3

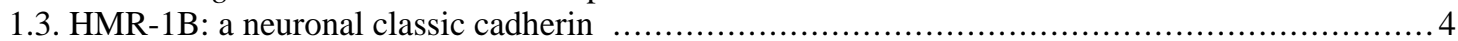

1.4. Classic cadherin function outside of the apical junction? ...................................... 4

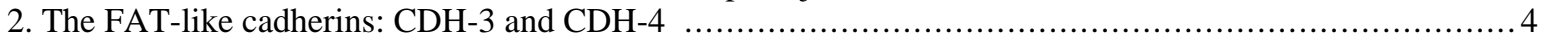

3. FMI-1, a $C$. elegans FLAMINGO/STAN cadherin ....................................................... 5

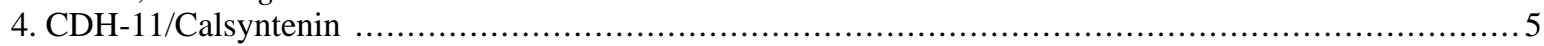

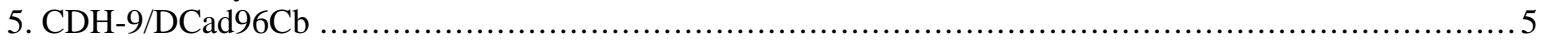

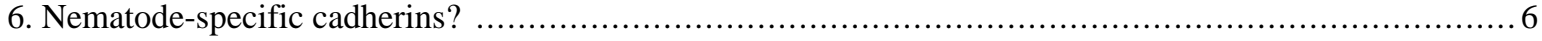

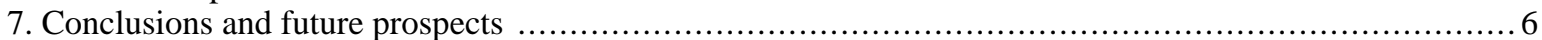

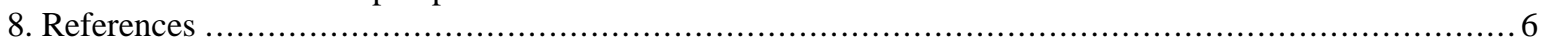

\begin{abstract}
The cadherins are a major class of membrane proteins with prominent roles in cell adhesion, and the regulation of tissue organisation and morphogenesis. The $C$. elegans genome encodes 13 cadherins, including representatives of the major cadherin sub-types that are conserved between insects and vertebrates: the so-called classic, Fat-like, Flamingo and calsyntenin classes. The function of most of these in $C$. elegans is still unknown, or poorly understood, mainly because clear loss-of-function mutations have been isolated for only a few. As is true for the cadherin families of other organisms, most is known about classic cadherin function. $C$. elegans has a single classic cadherin gene, which encodes two isoforms: one predominantly expressed in the nervous system, and the other more broadly expressed in all epithelial cells. The epithelial cadherin-catenin complex appears to be functionally equivalent to that found in Drosophila and vertebrates, and is critically required for embryonic morphogenesis. Mutant phenotypes have also been described for $c d h-3$ and $f m i-1$, which encode a Fat-like cadherin, and the $C$. elegans Flamingo homologue, respectively. $c d h-3$ mutants display incompletely penetrant defects in the morphogenesis of hyp10, the cell which forms the tip of the tail, and the excretory duct cell; though the mechanistic role of CDH-3 in these processes is not known. FMI-1 is required during neuronal development consistent with the known role of the Drosophila homologue in controlling tissue polarity. Five of the cadherins have no obvious homologues beyond the nematodes, and thus may be phyla-specific.
\end{abstract}

\footnotetext{
*Edited by Donald G. Moerman and James M. Kramer. Last revised March 31, 2005. Published December 29, 2005. This chapter should be cited as: Pettitt, J. The cadherin superfamily (December 29, 2005), WormBook, ed. The C. elegans Research Community, WormBook, doi/10.1895/wormbook.1.50.1, http://www.wormbook.org.

Copyright: (C) 2005 Jonathan Pettitt, This is an open-access article distributed under the terms of the Creative Commons Attribution License, which permits unrestricted use, distribution, and reproduction in any medium, provided the original author and source are credited

${ }^{\S}$ To whom correspondence should be addressed. E-mail: j.pettitt@abdn.ac.uk
} 


\section{The cadherin superfamily}

Cadherins are a superfamily of transmembrane proteins grouped by the presence of one or more cadherin repeats in their extracellular domains. Arrays of these approximately 110 residue domains form the intermolecular surfaces responsible for the formation of cadherin-mediated cell-cell interactions. Structural information from the analysis of several cadherin domains indicates that calcium ions bind at sites between adjacent cadherin repeats (CRs), forming a rigid rod (Patel et al., 2003). However, understanding of the mechanism by which this adhesion interface is formed comes primarily from studying the vertebrate classic cadherins. Given the structural diversity of the superfamily, it is unclear whether our model of cadherin function can be applied to all cadherins, and it seems likely that some members of the superfamily do not act as cell adhesion molecules.

C. elegans has 12 genes encoding 13 cadherins (Hill et al., 2001; Cox et al., 2004). Sequence similarity searches show that the same 12 genes, and no others, are present in the close relatives C. briggsae and $C$. remanei. Seven of these cadherins have homologues in non-nematode species and, with one exception (it lacks a member of the RET family of tyrosine kinases), C. elegans has representatives of all the main cadherin families that are conserved between Drosophila and vertebrates (Figure 1). Like Drosophila, it has no desmosomal cadherins (Garrod et al., 2002) or protocadherins (Frank and Kemler, 2002), these being vertebrate and chordate innovations, respectively.

\subsection{Classic cadherins and the cadherin-catenin complex}

The classic cadherins are by far the best understood in terms of both of mechanism and function within the context of animal development. The defining feature of this family is the presence of a conserved intracellular domain which mediates interactions with a set of cytoplasmic proteins termed catenins. On the basis of their extracellular domain organisation, these can be grouped into three sub-types. The extracellular domains of type I and II cadherins consist of five cadherin repeats (CRs); these two sub-types appear to be specific to the chordates. The type III cadherins have variable numbers of CRs and also contain a region termed the primitive classical cadherin domain (PCCD) which, together with variable numbers of EGF-like and laminin G repeats, lies between the CRs and the transmembrane helix. The PCCD is proteolytically cleaved during the maturation of Drosophila E-cadherin (Oda et al., 1999), and the conservation of this domain indicates that other type III classic cadherins may be similarly processed. Type III classic cadherins are found in both vertebrates and invertebrates (Oda et al., 2002; Tanabe et al., 2004), but are absent from mammals; they are the only classic cadherins found in the invertebrate groups studied to date.

The classic cadherin intracellular domain is a site for the assembly of a macromolecular complex that links the adhesion interface to the actin cytoskeleton. Two proteins are implicated in this activity: $\alpha$ - and $\beta$-catenin. $\beta$-catenin binds to both the $\mathrm{C}$-terminus of the cadherin intracellular domain and the $\mathrm{N}$-terminus of $\alpha$-catenin. $\alpha$-catenin binds to a number of proteins involved in actin binding, bundling and polymerisation, as well as binding directly to F-actin. Absence of $\alpha$ - or $\beta$-catenin results in defective cell adhesion and failure of cadherin-catenin complexes to associate with the actin cytoskeleton. A third protein, p120 catenin, binds to the classic cadherin intracellular domain at a site distinct from $\beta$-catenin. Classic cadherins together with the three catenins form a core functional unit, the cadherin-catenin complex (CCC), which is a major component of the apical junctions formed between epithelial cells. 


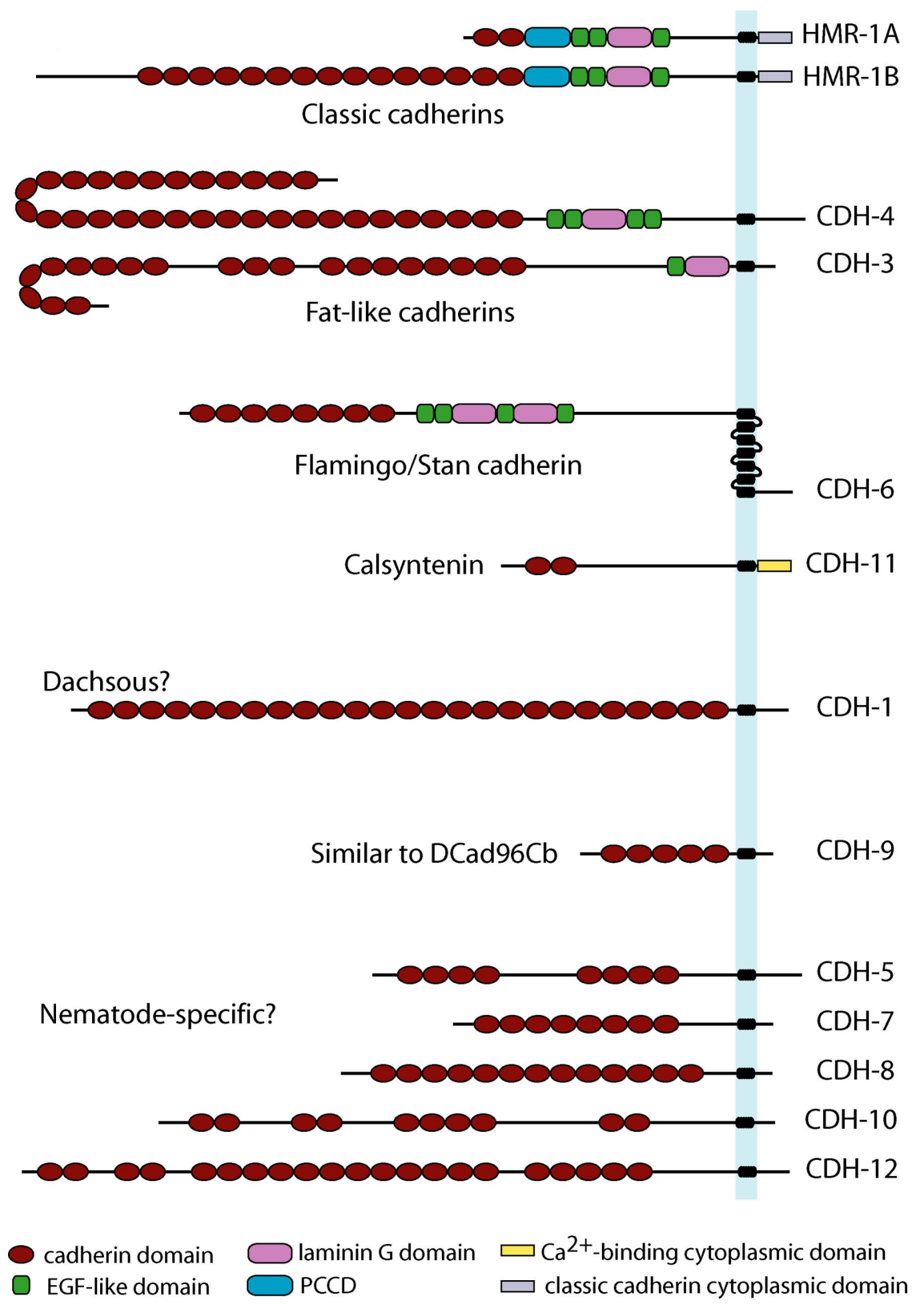

Figure 1. Structural diversity of the cadherin superfamily in . elegans. The 13 C. elegans cadherins are grouped according to their structural similarity with cadherins from other organisms. Each cadherin is positioned with its N-terminus to the left. PCCD = primitive classic cadherin domain (formerly termed non-chordate classic cadherin domain, it has also now been found in chordate classic cadherins).

\subsection{The C. elegans cadherin-catenin complex}

In contrast to vertebrates, but in common with Drosophila, $C$. elegans has single $\alpha-, \beta$ - and p120 catenins, encoded by hmp-1, hmp-2 and jac-1, respectively (Costa et al., 1998; Pettitt et al., 2003). It has a single classic cadherin gene, $h m r-1$, which encodes two proteins, HMR-1A and HMR-1B, via alternative splicing and alternative promoter use (Broadbent and Pettitt, 2002). HMR-1A is expressed in all epithelia plus an undefined set of neurons, while HMR-1B appears to be confined to neurons. Thus, C. elegans, like Drosophila, has both epithelial and neuronal classic cadherins; however, the mechanism by which they are generated appears unique to Caenorhabditis species.

As predicted on the basis of their sequence similarities, HMR-1A, HMP-1, HMP-2 and JAC-1 form a CCC that is a component of all apical junctions in C. elegans epithelia (Costa et al., 1998; Pettitt et al., 2003). jac-1 was 
identified solely on the basis of its sequence similarity to p120 catenins, whereas the other three genes were first defined on the basis of loss-of-function mutations that affect embryonic epidermal morphogenesis. Animals homozygous for hmp-1 or hmp-2 null mutations arrest during embryonic elongation with a characteristic Hmp ( Humpback) phenotype (Costa et al., 1998), whereas the majority of $h m r-1$ mutants show an earlier defect in ventral

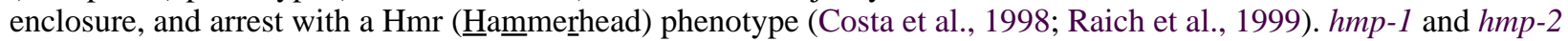
mutants don't show this defect because of maternal rescue: when both maternal and zygotic hmp-1/-2 function is removed by RNAi, affected embryos arrest with a Hmr phenotype (Costa et al., 1998; Raich et al., 1999). Thus, as in other organisms $\alpha$-catenin and $\beta$-catenin are essential for $C$. elegans classic cadherin function.

In contrast to the other catenins, JAC-1/p120 catenin is not essential for cadherin-mediated events in the $C$. elegans epidermis (Pettitt et al., 2003). However, it does appear to positively contribute to CCC function, since reducing its function enhances the phenotype of a weak hmp-l hypomorphic mutation. A similar situation exists for the sole Drosophila p120 catenin (Myster et al., 2003). In vertebrates however, p120 catenin appears to play a more critical function (Peifer and Yap, 2003; Fang et al., 2004), though even here its role in cadherin function does not appear to be as important as those of $\alpha$ - and $\beta$-catenin.

The most surprising aspect of the $C$. elegans cadherin-catenin complex is that it is dispensable for the formation and integrity of the major epithelia; cells of these tissues display apparently normal apical-basal polarity and, with few exceptions, remain tightly adherent to each other (Costa et al., 1998). This is in contrast to the severe defects in epithelial cell adhesion seen in Drosophila and vertebrates when cadherin-catenin complex function is reduced. The reason for this apparent discrepancy of classic cadherin function between C. elegans and other animals is not clear. It is noteworthy that regions of the Drosophila epidermis that do not undergo extensive morphogenetic events are tolerant of reduced classic cadherin function (Tepass et al., 1996).

\subsection{HMR-1B: a neuronal classic cadherin}

Functional analysis of the HMR-1B isoform indicates that classic cadherins also act during neuronal development in C. elegans. Animals with reduced or absent HMR-1B function are viable, but display incompletely penetrant defects in the guidance of the axons from a subset of motor neurons (Broadbent and Pettitt, 2002). This suggests that HMR-1B acts to maintain and/or stabilize the interactions between the axon growth cone and its substrate. However, since the penetrance of axonal guidance defects caused by loss of HMR-1B function is relatively low, it is likely that cadherin adhesion only augments other, more important, guidance cues.

\subsection{Classic cadherin function outside of the apical junction?}

HMR-1A, HMP-1 and HMP-2 co-localize to the regions of contact between all cells in the pre-morphogenetic embryo (the endogenous expression of JAC-1 is not known; Nance et al., 2003; Costa et al., 1998). This pattern of localisation at the basolateral surfaces of each embryonic blastomere suggests that the CCC has a function independent of its role at the apical junctions of epithelial cells. There is, however, no evidence which would implicate the CCC in events earlier than ventral enclosure, so either other adhesion molecules are able to compensate for the loss of classic cadherin function, or adhesion between cells of the early embryo does not involve cadherin function.

\section{The FAT-like cadherins: $\mathrm{CDH}-3$ and $\mathrm{CDH}-4$}

Two C. elegans cadherins, $\mathrm{CDH}-3$ and $\mathrm{CDH}-4$, have structural resemblances to the large Drosophila cadherin, Fat (Mahoney et al., 1991). Fat-like cadherins are also found in mammals (Dunne et al., 1995; Ponassi et al., 1999; Cox et al., 2000; Ciani et al., 2003; Mitsui et al., 2002; Nakayama et al., 2002), and these molecules all have extracellular domains that contain laminin-A globular domain and EGF-like repeats of the type found in type III classic cadherins. In addition, with the exception of CDH-3, all have similar numbers of cadherin repeats (33-34); a curious feature given that classic cadherins do not appear to show any trend in cadherin repeat number. However, there does not appear to be any similarity between the cytoplasmic domains of CDH-3 and CDH-4, nor any similarity to the cytoplasmic domains of Fat, or other Fat-like cadherins from Drosophila and mammals. In contrast, Drosophila Fat and the related Fat-like (Castillejo-Lopez et al., 2004) both share sequence motifs with mammalian Fat-like cadherins.

Recent work in Drosophila indicates that Fat is involved in the regulation of planar cell polarity (PCP; Rawls et al., 2002; Fanto et al., 2003; Strutt and Strutt, 2002; Casal et al., 2002; Ma et al., 2003; Yang et al., 2002) and studies of mammalian Fat1 demonstrate a role in the regulation of actin dynamics, in part through the recruitment of 
Ena/VASP proteins (Tanoue and Takeichi, 2004; Moeller et al., 2004). There is little functional information that would implicate either CDH-3 or CDH-4 in the regulation of cell polarity. Based upon GFP reporter fusion constructs, $c d h-3$ is expressed in subsets of epithelia and neurons (Pettitt et al., 1996). However, loss of $c d h-3$ function causes only variably penetrant defects in the morphogenesis of hyp10 and an impenetrant defect in excretory system function (Pettitt et al., 1996; L. Hodgson and J. Pettitt, unpublished), and the mechanistic cause of these defects is not known

There is even less information on the role played by CDH-4; RNAi does not give an obvious gross phenotype, and deletion mutants do not display any detectable defects in epidermal development (J. Pettitt, unpublished). $c d h-4$ promoter reporter constructs are expressed in the nerve ring and the axons of the ventral nerve cord (J. Pettitt, unpublished), so detailed analysis of axon guidance in these mutants may reveal a role for CDH-4.

\section{FMI-1, a C. elegans FLAMINGO/STAN cadherin}

fmi-1 (originally known as $c d h-6$ ) encodes the sole C. elegans homologue of the Drosophila Flamingo/Starry night cadherin; a seven-pass transmembrane protein that acts in the core planar cell polarity (PCP) pathway in Drosophila (Chae et al., 1999; Usui et al., 1999). Mutations affecting a mouse homologue result in failure of neural tube closure and defects in the polarity of the sensory hairs in the ear (Curtin et al., 2003); phenotypes which are indicative of defects in PCP signalling. Fmi/Stan is also required for axon guidance during development of the Drosophila visual system where it functions, at least in part, to polarise the growth cone actin cytoskeleton (though not involving other members of the PCP pathway; Lee et al., 2003; Senti et al., 2003). Thus Fmi/Stan cadherins in both mammals and flies act in the regulation of cell polarity, suggesting that this is a conserved function of this group of cadherins.

fmi-1 mutations recently identified from two independent genetic screens show phenotypes consistent with a role for FMI-1 in the regulation of cell polarity during neuronal development in C. elegans (G. Garriga and Y. Jin, personal communication). fmi- 1 mutants were identified based on aberrant HSN axon outgrowth and defective synaptogenesis. These mutants display abnormalities in the spacing and morphology of synapses formed by the VD and DD motor neurons, and they also display defects in the outgrowth of axons derived from these neurons. The HSN axon outgrowth phenotype can be phenocopied by fmi-1(RNAi), indicating that the HSN axon guidance defects arise though loss of fmi- 1 function. It will be interesting to determine whether FMI-1 function is confined to the nervous system, or whether other polarised cells are affected in fmi-l mutants.

\section{4. $\mathrm{CDH}-11 /$ Calsyntenin}

When the repertoire of $C$. elegans cadherins is compared to that of other organisms, the most striking sequence similarity matches are shown by CDH-11. Comparison of CDH-11 to its mammalian and Drosophila homologues shows that these proteins share significant sequence similarity along their entire lengths (Hill et al., 2001). The mammalian homologues of CDH-11 are termed calsyntenins, so-called because their cytoplasmic domains can bind synaptic calcium (Vogt et al., 2001). There is also evidence that they can associate with a protein complex involved in the processing of amyloid $\beta$-protein precursor (Araki et al., 2003). Calsyntenins are associated with the postsynaptic membranes of excitatory CNS synapses. The extracellular domains of calsyntenins are proteolytically cleaved close to the membrane, with the transmembrane-intracellular portion being internalised. This has led to a speculative model whereby calsyntenins modulate postsynaptic calcium levels. Based on strong sequence similarity, CDH-11 may play a similar role in C. elegans.

\section{5. $\mathrm{CDH}-9 / \mathrm{DCad} 96 \mathrm{Cb}$}

CDH-9 was initially thought to have no obvious sequence similarity to cadherins outside of C. elegans (Hill et al., 2001; Cox et al., 2004). However, reanalysing the comparisons between the cadherin repertoires of C. elegans and Drosophila reveals that Dcad96Cb is a putative homologue of CDH-9. Both cadherins have the same number of cadherin repeats, the first two CRs of CDH-9 show the highest match to the first two CRs of Dcad96Cb in BLAST searches of the non-redundant Genbank database, and although they have different sized cytoplasmic domains, both proteins terminate in the same four amino acid motif: TVYF, though the significance of this conservation is not known

Promoter-GFP fusion constructs derived from $c d h-9$ are expressed in the pharynx from the beginning of pharyngeal morphogenesis into adulthood (R. Babbar and J. Pettitt, unpublished). Confirming this result, $c d h-9$ was 
identified in a screen for genes expressed during pharyngeal development (Gaudet and Mango, 2002). This indicates that CDH-9 may have a role in the morphogenesis of the pharynx. However, $c d h-9(R N A i)$ has no obvious affect on pharyngeal morphology or behaviour (R. Babbar and J. Pettitt, unpublished).

\section{Nematode-specific cadherins?}

The remaining $C$. elegans cadherins do not have obvious homologues beyond the nematodes. One possible exception is CDH-1, which may be the C. elegans homologue of Drosophila Dachsous. This homology is tenuous as it is only based on the fact that they have similar numbers of CRs. However, their cytoplasmic domains are completely unrelated, and in the absence of any data regarding the function of $\mathrm{CDH}-1$, it is difficult to be certain of the relationship between these two cadherins. Recent work indicates that Dachsous, like Fat, acts in the PCP pathway in Drosophila (Lawrence et al., 2004; Matakatsu and Blair, 2004; Rodriguez, 2004), and it will thus be important to establish a function for $\mathrm{CDH}-1$ to determine if the superficial structural similarity between $\mathrm{CDH}-1$ and Dachsous has any functional basis.

CDH-8, and CDH-12 have obvious homologues only in C. briggsae and C. remanei. Homologues of CDH-5, -7 and -10 can also be detected in the more distantly related Brugia malayi. It seems likely that these cadherins are nematode-specific, but the alternative possibility, that homologues in non-nematode organisms exist, but have diverged significantly in structure, cannot be excluded. Functional analysis of these cadherins in comparison to similar studies of the Drosophila cadherins might help resolve this issue.

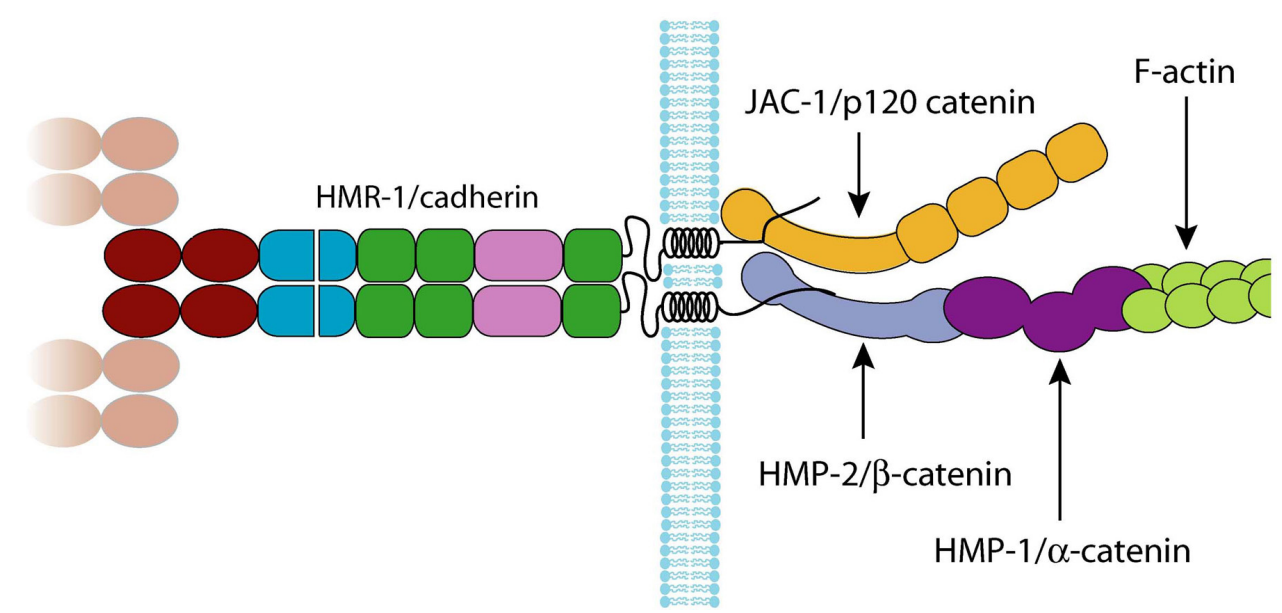

Figure 2. The organisation of the $\boldsymbol{C}$. elegans cadherin-catenin complex. HMR-1A is depicted as forming a dimer, with the N-terminal extracellular cadherin domains of the dimer interacting with those of HMR-1A dimers on opposing cell membranes. This is based on models of the cadherin adhesion interface derived from structural studies of vertebrate classic cadherins. It is not clear if these models hold for the type III classic cadherins. This diagram is by necessity a simplified view; there are many more proteins associated with the CCC, but the details of how they associate with the complex are not fully understood. The domains are identified using the key in (Figure 1). The PCCD is drawn to indicate the putative proteolytic cleavage site based upon sequence similarity to Drosophila E-cadherin.

\section{Conclusions and future prospects}

The repertoire of $C$. elegans cadherins is simple in comparison to that of vertebrates; the evolution of which involved the expansion and diversification of several cadherin subtypes. However, it is comparable to Drosophila, suggesting that the cadherin sub-types found in these two organisms are representative of those found in the last common ancestor of vertebrates, insects and nematodes. C. elegans is ideally suited to the dissection of cadherin function, but functional studies have been hampered by the paucity of observable loss-of-function phenotypes. Of the 12 cadherin genes, only hmr-l is essential for viability in the laboratory. However given that the expression of several cadherins is confined to the nervous system, it seems likely that loss-of-function phenotypes for some cadherins will be identified through the analysis of neuronal development, as has proven to be the case for fmi-1. Indeed lack of a severe phenotype may be an experimental advantage, since viable cadherin mutants would be more suited to detailed genetic analysis of their function. 


\section{References}

Araki, Y., Tomita, S., Yamaguchi, H., Miyagi, N., Sumioka, N., Kirino, Y., and Suzuki, T. (2003). Novel cadherin-related membrane proteins, alcadeins, enhance the X11-like protein-mediated stabilization of amyloid $\beta$-protein precursor metabolism. J. Biol. Chem. 278, 49448-49458. Abstract Article

Broadbent, I.D., and Pettitt, J. (2002). The C. elegans hmr-1 Gene Can Encode a Neuronal Classic Cadherin Involved in the Regulation of Axon Fasciculation. Curr. Biol. 12, 59-63. Abstract Article

Casal, J., Struhl, G., and Lawrence, P.A. (2002). Developmental compartments and planar polarity in Drosophila. Curr. Biol. 12, 1189-1198. Abstract Article

Castillejo-Lopez, C., Arias, W.M., and Baumgartner, S. (2004). The fat-like gene of Drosophila is the true orthologue of vertebrate fat cadherins and is involved in the formation of tubular organs. J. Biol. Chem. 279, 24034-24043. Abstract Article

Chae, J., Kim, M.J., Goo, J.H., Collier, S., Gubb, D., Charlton, J., Adler, P.N., and Park, W.J. (1999). The Drosophila tissue polarity gene starry night encodes a member of the protocadherin family. Development 126 , 5421-5429. Abstract

Ciani, L., Patel, A., Allen, N.D., and ffrench-Constant, C. (2003). Mice lacking the giant protocadherin mFAT1 exhibit renal slit junction abnormalities and a partially penetrant cyclopia and anophthalmia phenotype. Mol. Cell Biol. 23, 3575-3582. Abstract Article

Costa, M., Raich, W., Agbunag, C., Leung, B., Hardin, J., and Priess, J.R. (1998). A Putative Catenin-Cadherin System Mediates Morphogenesis of the Caenorhabditis elegans Embryo. J. Cell Biol. 141, 297-308. Abstract Article

Cox, B., Hadjantonakis, A.K., Collins, J.E., and Magee, A.I. (2000). Cloning and expression throughout mouse development of mfat1, a homologue of the Drosophila tumour suppressor gene fat. Dev. Dyn. 217, $233-240$. Abstract Article

Cox, E.A., Tuskey, C., and Hardin, J. (2004). Cell adhesion receptors in C. elegans. J. Cell Sci. 117, 1867-1870. Abstract Article

Curtin, J.A., Quint, E., Tsipouri, V., Arkell, R.M., Cattanach, B., Copp, A.J., Henderson, D.J., Spurr, N., Stanier, P., Fisher, E.M., Nolan, P.M., Steel, K.P., Brown, S.D., Gray, I.C., and Murdoch, J.N. (2003). Mutation of Celsr1 disrupts planar polarity of inner ear hair cells and causes severe neural tube defects in the mouse. Curr. Biol. 13, 1129-1133. Abstract Article

Dunne, J., Hanby, A.M., Poulsom, R., Jones, T.A., Sheer, D., Guo Chin, W., Ming Da, S., Zhao, Q., Beverley, P.C.L., and Owen, M.J. (1995). Molecular Cloning and Tissue Expression of FAT, the Human Homologue of the Drosophila fat Gene That Is Located on Chromosome 4q34-q35 and Encodes a Putative Adhesion Molecule. Genomics 30, 207-223. Abstract Abstract

Fang, X., Ji, H., Kim, S.W., Park, J.I., Vaught, T.G., Anastasiadis, P.Z., Ciesiolka, M., and McCrea, P.D. (2004). Vertebrate development requires ARVCF and p120 catenins and their interplay with RhoA and Rac. J. Cell Biol. 165, 87-98. Abstract Article

Fanto, M., Clayton, L., Meredith, J., Hardiman, K., Charroux, B., Kerridge, S., and McNeill, H. (2003). The tumor-suppressor and cell adhesion molecule Fat controls planar polarity via physical interactions with Atrophin, a transcriptional co-repressor. Development 130, 763-774. Abstract Article

Frank, M., and Kemler, R. (2002). Protocadherins. Curr. Opin. Cell Biol. 14, 557-562. Abstract Article

Gaudet, J., and Mango, S.E. (2002). Regulation of organogenesis by the Caenorhabditis elegans FoxA protein PHA-4. Science 295, 821-825. Abstract Article

Garrod, D.R., Merritt, A.J., and Nie, Z. (2002). Desmosomal cadherins. Curr. Opin. Cell Biol. 14, 537-545. Abstract Article 
Hill, E., Broadbent, I.D., Chothia, C., and Pettitt, J. (2001). Cadherin Superfamily Proteins in Caenorhabditis elegans and Drosophila melanogaster. J. Mol. Biol. 305, 1011-1024. Abstract Article

Lawrence, P.A., Casal, J., and Struhl, G. (2004). Cell interactions and planar polarity in the abdominal epidermis of Drosophila. Development 131, 4651-4664. Abstract Article

Lee, R.C., Clandinin, T.R., Lee, C.H., Chen, P.L., Meinertzhagen, I.A., and Zipursky, S.L. (2003). The protocadherin Flamingo is required for axon target selection in the Drosophila visual system. Nat. Neurosci. 6 , 557-563. Abstract Article

Ma, D., Yang, C.H., McNeill, H., Simon, M.A., and Axelrod, J.D. (2003). Fidelity in planar cell polarity signalling. Nature 421, 543-547. Abstract Article

Matakatsu, H., and Blair, S.S. (2004). Interactions between Fat and Dachsous and the regulation of planar cell polarity in the Drosophila wing. Development 131, 3785-3794. Abstract Article

Mitsui, K., Nakajima, D., Ohara, O., and Nakayama, M. (2002). Mammalian fat3: a large protein that contains multiple cadherin and EGF-like motifs. Biochem. Biophys. Res. Commun. 290, 1260-1266. Abstract Article

Moeller, M.J., Soofi, A., Braun, G.S., Li, X., Watzl, C., Kriz, W., and Holzman, L.B. (2004). Protocadherin FAT1 binds Ena/VASP proteins and is necessary for actin dynamics and cell polarization. EMBO J. 23, 3769-3779. Abstract Article

Myster, S.H., Cavallo, R., Anderson, C.T., Fox, D.T., and Peifer, M. (2003). Drosophila p120catenin plays a supporting role in cell adhesion but is not an essential adherens junction component. J. Cell Biol. 160, $433-449$. Abstract Article

Nakayama, M., Nakajima, D., Yoshimura, R., Endo, Y., and Ohara, O. (2002). MEGF1/fat2 proteins containing extraordinarily large extracellular domains are localized to thin parallel fibers of cerebellar granule cells. Mol. Cell Neurosci. 20, 563-578. Abstract Article

Nance, J., Munro, E.M., and Priess, J.R. (2003). C. elegans PAR-3 and PAR-6 are required for apicobasal asymmetries associated with cell adhesion and gastrulation. Development 130, 5339-5350.

Patel, S.D., Chen, C.S., Bahna, F., Honig, B., and Shapiro, L. (2003). Cadherin-mediated cell-cell adhesion: sticking together as a family. Curr. Opin. Struct. Biol. 13, 690-698. Abstract Article

Peifer, M., and Yap, A.S. (2003). Traffic control: p120-catenin acts as a gatekeeper to control the fate of classical cadherins in mammalian cells. J. Cell Biol. 163, 437-440. Abstract Article

Pettitt, J., Wood, W.B., and Plasterk, R.H. (1996). cdh-3, a gene encoding a member of the cadherin superfamily, functions in epithelial cell morphogenesis in Caenorhabditis elegans. Development 122, 4149-4157. Abstract

Pettitt, J., Cox, E.A., Broadbent, I.D., Flett, A., and Hardin, J. (2003). The Caenorhabditis elegans p120 catenin homologue, JAC-1, modulates cadherin-catenin function during epidermal morphogenesis. J. Cell Biol. 162, 15-22. Abstract Article

Ponassi, M., Jacques, T.S., Ciani, L., and ffrench-Constant, C. (1999). Expression of the rat homologue of the Drosophila fat tumour suppressor gene. MOD 80, 207-212. Abstract

Raich, W.B., Agbunag, C., and Hardin, J. (1999). Rapid epithelial-sheet sealing in the Caenorhabditis elegans embryo requires cadherin-dependent filopodial priming. Curr. Biol. 9, 1139-1146. Abstract Article

Rawls, A.S., Guinto, J.B., and Wolff, T. (2002). The cadherins fat and dachsous regulate dorsal/ventral signaling in the Drosophila eye. Curr. Biol. 12, 1021-1026. Abstract Article

Rodriguez, I. (2004). The Ena/VASP proteins and is necessary for actin dynamics and cell polarization. EMBO J. 23, 3769-3779. Article 
Rodriguez, I. (2004). The dachsous gene, a member of the cadherin family, is required for Wg-dependent pattern formation in the Drosophila wing disc. Development 131, 3195-3206. Abstract Article

Senti, K.A., Usui, T., Boucke, K., Greber, U., Uemura, T., and Dickson, B.J. (2003). Flamingo regulates R8 axon-axon and axon-target interactions in the Drosophila visual system. Curr. Biol. 13, 828-832. Abstract Article

Strutt, H., and Strutt, D. (2002). Nonautonomous planar polarity patterning in Drosophila: dishevelled-independent functions of frizzled. Dev. Cell 3, 851-863. Abstract Article

Tanoue, T., and Takeichi, M. (2004). Mammalian Fat1 cadherin regulates actin dynamics and cell-cell contact. J. Cell Biol. 165, 517-528. Abstract Article

Tepass, U., Gruszynski-DeFeo, E., Haag, T.A., Omatyar, L., Torok, T., and Hartenstein, V. (1996). shotgun encodes Drosophila E-cadherin and is preferentially required during cell rearrangement in the neurectoderm and other morphogenetically active epithelia. Genes Dev. 10, 672-685. Abstract

Usui, T., Shima, Y., Shimada, Y., Hirano, S., Burgess, R.W., Schwarz, T.L., Takeichi, M., and Uemura, T. (1999). Flamingo, a Seven-Pass Transmembrane Cadherin, Regulates Planar Cell Polarity under the Control of Frizzled. Cell 98, 585-595. Abstract Article

Vogt, L., Schrimpf, S.P., Meskenaite, V., Frischknecht, R., Kinter, J., Leone, D.P., Ziegler, U., and Sonderegger, P. (2001). Calsyntenin-1, a proteolytically processed postsynaptic membrane protein with a cytoplasmic calcium-binding domain. Molecular and Cellular Neuroscience 17, 151-166. Abstract Article

All WormBook content, except where otherwise noted, is licensed under a Creative Commons Attribution License 